\title{
Hyperspectral Imagery Further Unmixing Based On Analysis Of Variance
}

\author{
Wang Lei ${ }^{1,2} *$ \\ 1 State key Laboratory for Information Engineering in \\ Surveying, Mapping and Remote Sensing \\ 2 Collaborative Innovation Center for Geospatial \\ Technology \\ Wuhan University \\ Wuhan, China \\ wlei@whu.edu.cn \\ * Corresponding Author
}

\author{
Shao Zhenfeng ${ }^{1,2}$ \\ 1 State key Laboratory for Information Engineering in \\ Surveying, Mapping and Remote Sensing \\ 2 Collaborative Innovation Center for Geospatial \\ Technology \\ Wuhan University \\ Wuhan, China \\ shaozhenfeng@whu.edu.cn
}

\begin{abstract}
Hyperspectral imagery unmixing model based on sparse regression uses the existing endmembers' library as priori information. Usually, the existing endmembers' library contains almost all kinds of ground objects. Even though sparse regression-based imagery unmixing method added sparse constraint to the original unmxing model, the solution is still far away as sparse as real scenario. Therefore, we propose a hyperspectral imagery further unmixing method based on the analysis of variance. In this method, fractional abundances unmixed by sparse regression-based approach are analyzed with t-test. If the fractional abundances are not significant enough, the corresponding endmembers will be removed and a new optimal endmember subset will be extracted. Then the unmixing process was redid with acquired optimal endmember subset and the final result will be acquired. The experimental results indicate that the proposed method could acquire sparser solution, which is closer to the real sparsity of abundance, both in simulate scenario and real scenario. Furthermore, the precision of the endmember recognition of proposed method is more than $97 \%$, which is a pretty good result.
\end{abstract}

Keywords-Hyperspectral imagery; Linear unmixing; Sparse regression; Analysis of variance

\section{INTRODUCTION}

Hyperspectral remote sensing imagery with higher spectral resolution and abundant spectral information of all kinds of objects, has been widely used for ground objects classification, anomaly detection, targets recognition and ground objects analysis (Jiang, X., et al. 2014; Yu, X., et al. 1997; Pu, Gong. 2000; Li. 2011), etc. Nevertheless, hyperspectral imagery mixed pixel often includes many different kinds of ground objects, which will hinder the further analysis of remote sensing imagery, so the unmixing of hyperspectral imagery is very important not only to recognize the different kinds of ground objects, extract their characteristic spectrum, but also to estimate the proportion of different contained ground objects, analyze the spectrum information and the content information. Hyperspectral imagery unmixing has crucial position in analysis of hyperspectral remote sensing imagery, the unmixed result is relate with application directly.

There are two kinds of hyperspectral imagery mixing models: linear mixing model and nonlinear mixing model.
Linear mixing model assumes that the observed spectral signature can be expressed in the form of linear combination of a number of spectral signatures of ground objects contained in the mixed pixel, the abundances of endmembers are just the coefficients of the linear combination. Conversely, in nonlinear mixing model, the apparent albedo of the mixed pixel is a nonlinear function of the albedos of the individual endmembers. As linear mixing model has simple expression, explicit physical meaning and strong universality, it has been applied widely in imagery unmixing (Keshava N, Mustard J F. 2002). In this paper, we focus on the linear mixing model.

The primary task of mixed pixel decomposition is to extract spectral signature of the pure ground object, which also be called endmember extraction. In hyperspectral remote sensing imagery stage, each mixed pixel usually contains 2 or more kinds of ground objects, and the number of endmembers contained in the whole imagery is often less than 20. However, it's very difficult to extract the contained endmembers and their spectral information in the whole imagery. In recent years, as there are more and more building and opening of ground object' spectral library, such as the mineral spectral library of Unite States Geological Survey and the ASTER spectral library of National Aeronautics and Space Administration, it's available to use the existing ground objects' spectral library to decompose the mixed pixels instead of extracting endmembers from the imagery directly, which can avoid the influence of the errors produced during the process of extracting endmembers from the original imagery. There are hundreds and thousands of endmembers in the spectral library. However, when we decompose mixed pixels with the existing spectral library, there are only several active endmembers actually. That's to say, when we express the spectral signature of the mixed pixel with linear combination of the endmembers in the existing endmembers spectral library, most of the coefficients are zero, which is named the sparsity of linear unmixing of the mixed pixels.

In view of the sparsity of linear expression of the mixed pixels with existing spectral library, sparse regressionbased method has been introduced to linear mixing model and has acquired good effect (Bioucas-Dias J. M. et al. 2012). Hyperspectral imagery unmixing method based on sparse regression is a kind of semi-supervised unmixing 
method, which uses the existing endmembers spectral library as prior information, assumes that the existing spectral library contains all kinds of endmembers in the whole imagery and the mixed pixel could be expressed as the linear combination of some endmembers. It emphasizes the sparsity of unmixed abundance, requires that the umixed abundance vectors should be as sparse as possible when the reconstruction error is controllable, so that the final result is sparse and close to the real scenario. There are many ways to solve the sparse regression-based imagery unmixing model, such as orthogonal matching pursuit algorithm (Pati Y. C., Rezahfar R., Krishnaprasad P. 2003), basis pursuit algorhthm (Chen S., D. Donoho, and M. Saunders. 2001), iterative spectral mixture analysis method (Rogge D. M., B. Rivard, J. Zhang, and J. Feng. 2006), SUnSAL (Bioucas-dias J. M., and M. Figueiredo. 2010) and its improved algorithm, etc. Numerous experimental results state that the unmixed result obtained by SUnSAL and its improved algorithm perform better in imagery unmixing.

Though sparse regression based unmixing model emphasizes adds sparse constraint to the unmixed model, the spasity of unmixed abundance is still far more than the sparsity of real scenario because of observation noise, endmember variability, algorithm inaccuracies, environmental conditions and so on. In other words, the number of endnumbers contained in the unmixed result is still far more than the number of the endmembers contained in the unmixed pixel. Nevertheless, some endmembers in the unmixed result are not such significant enough to the whole solution, they are not the endmembers of the mixed pixel but the error portion caused by noise. In this paper, abundances unmixed by sparse regression based method are analyzed further. If the abundances are not significant enough, the corresponding endmembers will be removed and a new optimal endmember subset will be extracted. Then the unmixing process was redid with obtained optimal endmember subset and the sparser and more accurate unmixed solution was acquired.

\section{MIXED PIXEL DECOMPOSITION MODEL}

Linear spectral mixing model can be expressed as

$$
\mathbf{y}=\mathrm{Dx}+\boldsymbol{\varepsilon}
$$

Where $y$ is a column vector with $\mathrm{L}$ ( $\mathrm{L}$ denotes the number of spectral bands) dimensions, represents the observed spectral reflectance of mixed pixel. $\mathrm{D}$ is a $\mathrm{L} \times \mathrm{M}$ ( $\mathrm{M}$ denotes the number of endmembers) endmember matrix, each column of $\mathbf{D}$ denotes a characteristic spectrum of endmember. $\mathbf{x}$ is a column vector with $\mathbf{M}$ dimensions, denotes the proportion of the corresponding endmembers. $\boldsymbol{E}$ denotes the Gaussian white noise or model error with $\mathrm{L}$ dimensions.

Assume that there are $\mathrm{N}$ pixels in the hyperspectral remote sensing imagery. Thus, the original expression of linear spectral mixing model could be expanded as:

$$
\mathbf{Y}=\mathbf{D X}+\mathbf{E}
$$

Where $Y \in R^{L \times N}$, each column of it is an observed spectrum of the pixel. $\mathbf{X} \in R^{M \times N}$ denotes the abundance matrix, each column of it is the corresponding abundance. $\mathbf{E} \in R^{L \times N}$ is the error matrix.
In linear mixing model, abundance matrix $\mathbf{X}$ has explicit physical meaning. It has to satisfy two constraints: nonnegative constraint

$$
\mathrm{X}_{\mathrm{i} j} \geq 0, i=1,2 \cdots M_{x} j=1,2 \cdots N
$$

and sum-to-one constraint

$$
\sum_{i=1}^{M} \mathbf{X}_{i j}=1, j=1,2 \cdots N
$$

When endmember matrix $\mathrm{D}$ belongs to $R^{L \times N}(\mathrm{~L} \times \mathrm{M})$, the solution of abundance vector $\mathbf{x}$ is not unique. However, because of the sparsity of linear unmixing of the mixed pixels, above problem could be transformed to find the sparsest (has least nonzero element) abundance vector $\mathbf{x}$. This optimization problem could be denoted as:

$$
\begin{gathered}
\underset{\mathbf{x}}{\operatorname{Min}}\|\mathbf{x}\| \|_{0} \\
\text { subject to }\|\mathbf{y}-\mathbf{D x}\| \mid \leq \varepsilon_{0}, \mathbf{x} \geq \mathbf{0} \text {, and } \mathbf{1}^{T} \mathbf{x}=1
\end{gathered}
$$

Where $\|\mathbf{x}\|_{0}$ is the $l_{0}$ norm of vector $\mathbf{x}$, denotes the number of nonzero elements in vector $\mathbf{x} . \varepsilon_{0}$ is the error tolerance due to noise and modeling errors. Assume a while for that $\varepsilon_{0}=0$, if the equation $y=D x$ has a solution satisfying 2|| $\mathbf{x} \|_{0} \leq$ spark (D), where spark (D) is the smallest number of columns of $\mathbf{D}$ that are linearly dependent, it is necessarily the unique solution of (5). For $\varepsilon_{0}>0$, the notion of uniqueness is replaced with the notion of stability.

In most real situations, we do have 2|| $\mathbf{x} \|_{0} \leq \operatorname{spark}$ (D) and consequently, at least in noiseless situations, the solutions of (5) are unique. But, the optimization of formula (5) is a NP-hard problem. There is no way to solve it in a straightforward way, greedy algorithms are often needed to solve this problem. However, Tao, Candes and Donoho et al have proved that when the signal is sparse enough, $l_{0}$ can be transformed to $l_{1}$ norm. Therefore, above optimization problem could be transform to

$$
\min _{\mathbf{x}}\|\mathbf{x}\|_{1} \text { subject to }\|\mathbf{y}-\mathrm{Dx}\| \leq \varepsilon_{0}, \mathbf{x} \geq \mathbf{0}
$$

An equivalent formulation to (5) is

$$
\begin{gathered}
\left.\min _{\mathbf{x}} \frac{1}{2}\|\mathbf{y}-\mathrm{Dx}\|\right|_{2} ^{2}+\lambda|\|\mathbf{x}\||_{1} \\
\text { subject to }\|\mathbf{y}-\mathrm{Dx}\| \leq \leq \varepsilon_{0}, \mathbf{x} \geq \mathbf{0}
\end{gathered}
$$

Where $\lambda$ is a regularization parameter, which is related with the Lagrange multiplier of the inequality $\|y-D x\| \leq \varepsilon_{0}$. In real situation, because of the distribution of observation noise, endmember variability and environmental conditions, the sum of abundance is difficult to be one exactly. Optimization problem (6) is a convex optimization problem, which could be solved with optimization algorithm. Numerous experimental results indicate that $\mathrm{SUnSAl}$ algorithm is better to solve above convex optimization problem. Nevertheless, no matter which algorithm we choose to solve the above problem, the unmixed abundance is still far away as sparse as real scenario. 


\section{FURTHER IMAGERY UNMIXING WITH ANALYSIS OF VARIANCE (ANOVA)}

In view of the disadvantages of existing algorithms in linear unmixing model, Wang and Shao (2014) proposed the hyperspectral imagery unmixing method based on sparse endmember subset. In this method, unmxing algorithm based on sparse regression was used to unmix the original imagery, the unmixed abundance sorted in descending order was denoted as $\bar{x}=\left(x_{1,}, x_{2, \ldots}, x_{M}\right)$, and the series $\left\{c_{i}\right\}$ are acquired according to

$$
d_{i}=x_{i}-x_{i+1}, c_{i}=1-d_{i} / d_{i+1} \text {, for } i=1, \ldots, k
$$

The sequence number of the max element in series $\left\{c_{\mathrm{i}}\right\}$ was found, and the endmembers before the sequence number were regard as significant and used to replace the original endmembers to acquire the sparse endmember subset. The unmixing was redid with obtained sparse endmember subset and the final unmixed result was acquired.

In above method, the extraction of the sparse endmember subset is only depends on the value of unmixed abundance. While the value of abundance (or coefficient) is not such associate with the significance of linear relationship between the endmember's characteristic spectra (independent variable) and the observed spectra (dependent variable). Therefore, the above method is easier to remove the endmembers contained in the mixed pixels during the process of extracting sparse endmember subset. Wu (2007), Li (2011) et al have analyzed the influence of missing endmembers and chose endmembers to the accuracy of umixed result: When too much endmembers were chose, the unmixed abundance was still the unbiased estimation of the real abundance; While if some kinds of endmembers were removed by mistake, the unmixed abundance will no longer be the unbiased estimation of the real abundance, and the reconstruct error will be larger. On one hand, we hope the unmixed result as sparse as possible. On the other hand, it will make more contained endmembers missed if the result is sparse too much, and the umixed abundance will no longer be the unbiased estimation of the real abundance.

Based on above analysis, this paper proposed a hyperspectral imagery unmixing method based on analysis of variance (ANOVA). In this method, the original imagery was unmixed with SUnSAL algorithm first. Then, the significance of the unmixed equation was estimated with analysis of variance and the significance of the unmixed abundances (coefficients of the unmixed equation) were tested at the same time. The endmembers corresponding to the unmixed abundance, which is not significant enough, will be removed and the optimal expression endmember subset will be acquired. Lastly, the imagery was further unmixed with obtained optimal expression endmember subset, and the sparser and more accurate unmixed result will be acquired. The process of hyperspectral imagery further unmixing based on analysis of variance (ANOVA) consists of 4 steps:

\section{Hyperspectral imagery further unmixing based on}

\section{ANOVA:}

step1. The original imagery was unmixed with SUnSAL algorithm first;

step2. A new endmembers subset $\mathbf{A}_{s}=\left[\boldsymbol{a}_{1}, \boldsymbol{a}_{2, \ldots,} \boldsymbol{a}_{m}\right]$ was built with the endmembers whose abundance is nonzero. Then the optimization problem

$$
\left.\min _{\mathrm{x}} \frac{1}{2}\|\mathrm{y}-\mathrm{Dx}\|\right|_{2} ^{2} \text { subject to } \mathbf{x} \geq 0
$$

will be solved. The cardinal number of the endmembers subset is sparse enough and it's not necessary to add the sparse constraint to the model now. The unmixed coefficient vector $\mathbf{x}$ is just the endmembers' abundances;

Step3. Each coefficient of the unmixed equation $y=x_{1} a_{1}+x_{2} a_{2}+\cdots+x_{m} a_{m}$ obtained from step2 was tested with t-test. If p-value of t-test is larger than default threshold $\alpha=0.05$, the coefficient is regard as not significant enough. The endmembers whose abundance is not significant enough were removed from the endmembers subset $\mathbf{A}_{s}$ to obtain a new endmembers subset $\mathbf{A}_{s}^{v}$;

Step4. Redo step2 with endmembers subset $\boldsymbol{A}_{s}$ acquired from setp3 and obtain a new unmixed equation $y=x_{1} a_{1}+x_{2} a_{2}+\cdots+x_{m} a_{m}$. The significance of the unmixed equation was with F-test. If the pvalue of F-test is larger than threshold 0.05 , the unmixed result is regard as reliable and to be the final result. While if the p-value of F-test is smaller than threshold 0.05 , the unmixed result is not significant enough. In other words, too much endmembers contained in the mixed pixel have been removed. Let $\alpha=\rho \alpha \quad(\rho>1$ is the increasing coefficient of $\alpha, \alpha$ should not larger than 1), restart step3.

\section{ESTIMATION OF IMAGERY UNMIXING RESULT}

In order to estimate the imagery unmixed result thoroughly, this paper estimates the unmixed result from 5 aspects: accuracy of endmember recognition, missing rate of endmember recognition, sparsity of unmixed abundance, ratio of the number of pixels whose sum of abundance fall in the confidence interval and the total number of pixels in the whole imagery, and p-value of normal distribution test of the reconstruction error. In the experiment with real data, only the last three indicators are available as the real abundance was unknown.

\section{A. Accuracy of endmember recognition}

Usually, unmixed endmembers are not completely same as truly contained endmembers. Some endmembers are truly contained in the unmixed pixel but not be recognized by unmixing algorithm. While some unmixed endmembers are not the endmembers contained in the unmixed pixel, they are called pseudo endmembers. Let $k$ be the spasity of unmixed abundance, denotes the total 
number of endmembers recognized by unmixing algorithm. Let $k_{t}$ denotes the number of the recognized endmembers which were truly contained in the unmixed pixel. Denote $R_{p}=k_{t} / k$ as the accuracy of endmember recognition. Higher accuracy means better unmixed result.

\section{B. Missing rate of endmember recognition}

Let $k_{\text {miss }}$ denotes the number of endmembers which are truly contained in the unmixed pixel but not be recognized by unmixing algorithm, $k_{\text {true }}$ is the number of endmembers truly contained in the unmixed pixel. Missing rate

$$
R_{\text {migs }}=k_{\text {miss }} / k_{\text {true }}
$$

decides whether the unmixed abundance is the unbiased estimation of the real abundance or not. The lower the missing rate is, the better the ummixed result will be.

\section{Sparsity of unmixed abundance}

The number of endmembers in endmember spectral library is far more than the number of endmembers contained in one unmixed pixel. Though sparsity-based unmixing algorithm adds sparse constraint to the original model, the solution is still not as sparse as real abundance. The sparsity of unmixed result is the most important characteristics of imagery unmixing. The more sparsity $k=\|\hat{\mathbf{x}}\|_{\text {o }}$ ( $\hat{\mathbf{x}}$ denotes the unmixed abundance vector) close to the real sparsity of the unmixed pixel (Generally speaking, the real sparsity of unmixed pixel is $4 \sim 5$ ), the better the result was.

\section{Ratio of the number of pixels whose sum of abundance fall in the confidence interval and the total number of pixels}

As the physical meaning of abundance, the sum of unmixed abundance should be equal to one. However, because of the interference of noise and the influence of algorithm inaccuracies, the unmixed abundance often not equals to one in real situation. Considered from this aspect, if the sum of unmixed abundance is closer to one, it could indicate that the unmixed result is better. Let $n_{\text {in }}$ denotes the number of pixels fall in the confidence interval $\left[S_{u m}{ }_{d x} S_{u m}\right], n$ represents the total number of pixels. Then

$$
R_{\text {sum }}=n_{\text {in }} / n
$$

is the ratio of the number of pixels whose sum of abundance fall in the confidence interval and the total number of pixels. The more $S u m_{d}$ and $S u m_{u}$ close to 1 , and the larger the value of $R_{\text {sum }}$ is, the better the unmixed result will be.

\section{E. p-value of normal distribution test of the reconstruction error}

Reconstruction error should be Gaussian white noise satisfy normal distribution. Therefore, in this paper, we proposed to use p-value of normal distribution test of the reconstruction error as an indicator to estimate the unmixed result. Denote $p_{i}$ as $p$-value of the normal distribution test of the ith pixel's reconstruction error, $\mathrm{n}$ is the total number of pixels. Then

$$
p_{\text {norm }}=\sum_{i=1}^{n} p_{i} / n
$$

is the average $\mathrm{p}$-value. The more $p_{\text {norm }}$ close to 1 , the more likely the reconstruction error obey normal distribution, and the better the result will be.

\section{EXPERIMENTS AND ANALYSIS}

In order to demonstrate the effectiveness of proposed method, two experiments were designed in this paper: experiment with simulate data and experiment with real data. In the simulate scenario, the real abundance was known, so all the indicators could be used to estimate the unmixed result. Conversely, in the experiment with real data, the real abundance was unknown, and only the last three indicators were available. In particular, the sparsity of the unmixed result is the most important aspect to evaluate the unmixed result.

\section{Experiments and analysis with simulate data}

\section{1) Simulate data constructionSelection}

In this experiment, 498 endmembers from the mineral spectral library of AVIRIS cuprite remote sensing imagery dataset of Nevada, United States were extracted to construct the simulate endmember library. The simulate dataset includes 1000 mixed pixels. Usually, in hyperspectral remote sensing imagery, the sparsity of unmixed pixel is 4 or 5 (Iordache M. D., Bioucas-Dias J. M., Plaza A.2010), so the sparsity in this simulate data was designed to be 4 . Each pixel is the linear combination of 4 endmembers chose randomly from the spectral library according to equation (1). The corresponding abundances are chose from interval $[0,1]$ randomly and satisfy the constraint of nonnegative and sum-to-one. Then the Gaussian white noise with $20 \mathrm{db}, 40 \mathrm{db}, 60 \mathrm{db}$ were added to them respectively.

\section{2) Simulate data constructionSelection}

The simulate data was unmixed with SUnSAL algorithm first, then the obtained initial result was further unmixed according to the step of imagery further unmixing method based on ANOVA in 3. During the unmixing process, we choose $\lambda=10^{-4}$, which has been demonstrated to be a better one, as the regularization parameter. The increasing coefficient of $\alpha$ in step 4 of ANOVA-based imagery further unmixing method was set to be $\rho=1.2$, where the more the value of $\rho$ close to 1 , the more accurate the result will be, but it will also cost more iterative steps to solve the problem.

Table 1 depicts the accuracy $R_{p}$, missing rate $R_{\text {miss }}$, ratio of the number of pixels whose sum of abundance fall in the confidence interval and the total number of pixels in the whole imagery $R_{\text {sum }}$, sparsity and p-value of normal distribution test of the reconstruction error pnom of the unmixed result solved by proposed method, sparse endmembers subset based method (SES) and SUnSAL algorithm respectively when $\lambda=10^{-4}$ and $\rho=1.2$. Table 1 shows that, compared with the other two algorithms, the proposed method could recognize the endmembers contained in the unmixed pixel more 
precisely, the sparsity of unmixed abundance is closer to the real sparsity 4 with any level of noise interference. When the SNR is small, the proposed method still could recognize the contained endmembers precisely, which can acquire very high accuracy, while the missing rate will also be increased, the noise will make more interference to the proposed algorithm.

Table 1. Quantitative evaluation of the unmixed results with simulate data

\begin{tabular}{c|c|c|c|c|c}
\hline \hline & $R_{\text {miss }}$ & $R_{p}$ & $R_{\text {sum }}$ & Sparsity & $P_{\text {norm }}$ \\
\hline Proposed algorithm(SNR=20) & 0.47 & 0.9725 & 0.48 & 2.18 & 0.7062 \\
\hline SES algorithm(SNR=20) & 0.2575 & 0.3626 & 0.69 & 8.19 & 0.7891 \\
\hline SUnSAL(SNR=20) & 0.1975 & 0.2346 & 0.18 & 13.68 & 0.7759 \\
\hline Proposed algorithm (SNR=40) & 0.075 & 1 & 1 & 3.7 & 0.7542 \\
\hline SES algorithm (SNR=40) & 0.08 & 0.4381 & 1 & 8.4 & 0.7682 \\
\hline SUnSAL(SNR=40) & 0.0375 & 0.2659 & 1 & 14.48 & 0.7653 \\
\hline Proposed algorithm (SNR=60) & 0.0275 & 0.9873 & 1 & 3.94 & 0.7432 \\
\hline SES algorithm (SNR=60) & 0.0625 & 0.4331 & 0.99 & 8.66 & 0.6981 \\
\hline SUnSAL(SNR=60) & 0.0225 & 0.3174 & 1 & 12.32 & 0.7056 \\
\hline \hline
\end{tabular}

\section{B. Experiment and analysis with real data}

\section{1) Experiment data}

The real data used in this paper is the AVIRIS cuprite remote sensing imagery dataset of Nevada, United States. This airborne hyperspectral imagery dataset includes 224 spectral bands, 498 characteristic spectra of endmembers. It has been widely used to verify the validity of endmember extraction and imagery unmixing.

\section{2) Simulate data constructionSelection}

The original imagery was unmixed with SUnSAL algorithm first with the regularization parameter was set to be $\lambda=10^{-4}$ and the termination threshold was set to be $\delta=10^{-4}$. Then the obtained initial result was further unmixed according to the step of imagery further unmixing method based on ANOVA in 3. The increasing coefficient of $\alpha$ in step4 was set to be $\rho=1.2$. [0.8,1.2] was chose as the confidence interval (Li.2011). Lastly, the problem was solved with acquired optimal endmember subset and the solution was compared with the result obtained by SUnSAL algorithm and sparse endmembers subset based method.

Table 2 shows the ratio of the number of pixels whose sum of abundance fall in the confidence interval and the total number of pixels in the whole imagery $R_{\text {sum }}$, sparsity of the unmixed abundance and p-value of normal distribution test of the reconstruction error pnorm of the unmixed result acquired by proposed method, sparse endmembers subset based method (SES) and SUnSAL algorithm respectively. Table 2 demonstrates that, compared with other two kinds of algorithms, when $R_{\text {sum }}$ is basically equal, the sparsity of unmixed abundance obtained by proposed method is closer to the real sparsity (4 5), and the reconstruct error is more like to obey normal distribution.

Table 2. Quantitative evaluation of the unmixed results with real hyperspectral imagery

\begin{tabular}{c|c|c|c}
\hline \hline & $R_{\text {sum }}$ & Sparsity & $P_{\text {norm }}$ \\
\hline Proposed algorithm & 0.8089 & 5.0978 & 0.1180 \\
\hline SES algorithm & 0.8294 & 8.9447 & 0.0686 \\
\hline SUnSAL algorithm & 0.8613 & 17.9866 & 0.088 \\
\hline \hline
\end{tabular}

In Figure 1, we focus on two minerals: Alunite and Buddingtonite. Figure 1 (a) is the classification figure of the two minerals. The Figure of classification is a binary imagery. Figure 1 (b), (c) and (d) show the figure of unmixed abundance acquired by proposed method, sparse endmember subset based method and SUnSAL algorithm respectively. Figure1 and Table2 demonstrate that the proposed method could acquire sparser result than SUnSAL algorithm, and keep the significant endmembers more effectively than sparse endmember subset based method. 

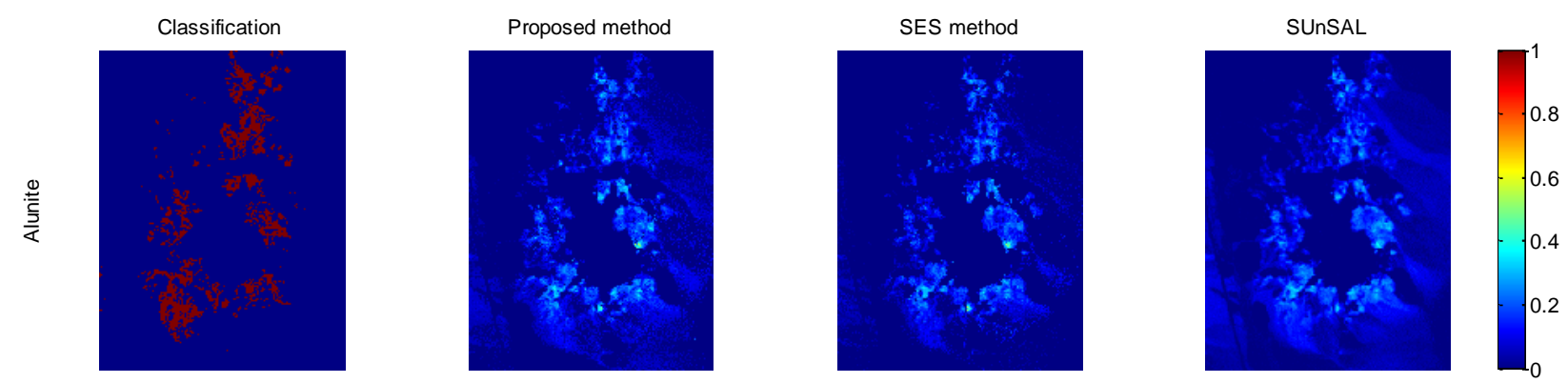

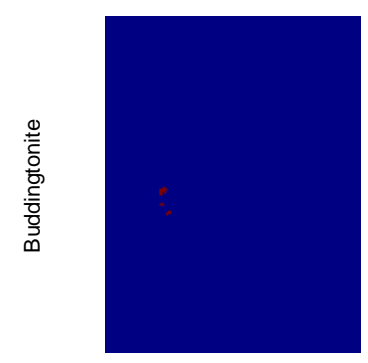

(a)

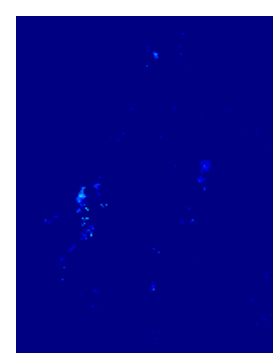

(b)

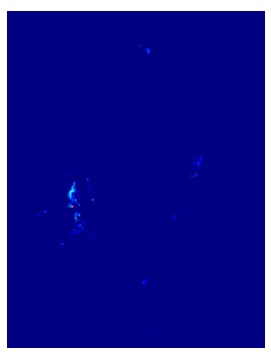

(c)

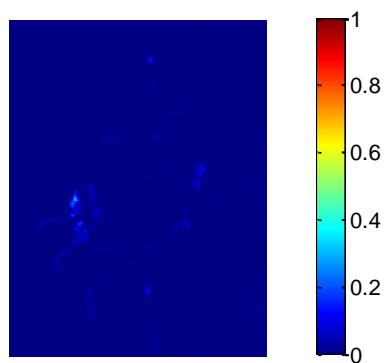

(d)

Figure 1. (a) Classification figure of the two minerals; (b), (c) and (d) are the distribution of the unmixed abundance of two minerals acquired by proposed method, SES algorithm and SUnSAL algorithm respectively

\section{CONCLUSIONS}

It is difficult to balance the precision and sparsity of unmixed abundance to acquire good result at the same time. Emphasizing the precision of the unmixed result will led the sparsity of unmixed abundance to be far away as sparse as the real abundance. However, when we pay more attention to the sparsity of the unmixed abundance, the precision of the unmixed result will not be such good. The proposed method analyzed the initial unmixed abundance to obtain the optimal expression endmember subset according to the significant test, so that the imagery can be further unmixed with acquired optimal endmember subset. Compared with SUnSAL algorithm and sparse endmember subset based unmixing method, the proposed method could acquire sparser and more accurate abundance.

\section{ACKNOWLEDGMENT}

This research was financially supported by the National Science \& Technology Specific Projects under Grant 2012YQ16018505, 2013BAH42F03, by National Natural Science Foundation of China under Grant 61172174, the Basic Research Program of Hubei Province(2013CFA024), Special Project on the Integration of Industry, Education and Research of Guangdong Province (2012B090500016), Shenzhen science and Technology Development Foundation (JCYJ20120618162928009), public research fund on surveying and mapping (201412010).

\section{REFERENCES}

[1] Yu, X., et al. Generalized linear feature detection of weak targets in spectrally mixed clutter[C]. Conference on Signal and Data Processing of Small Targets. Sandiego: Proceedings of the society of photo-optical instrumentation engineers (SPIE), 1997, 3163: 108-116.

[2] Jiang, X., et al. Quantitative Interpretation of Mineral Hyperspectral Images Based on Principal Component Analysis and Independent Component Analysis Methods[J]. Applied Spectroscopy, 2014, 68(4): 502-509.

[3] LI Ersen, XU Bo, LI Na, ZHOU Xiaoming. Minimum Volume Constrained Linear Spectral Unmixing Algorithm[J]. Geomatics and Information Science of Whuhan University, 2011, 36(6): 683685.

[4] Keshava N, Mustard J F. Spectral Unmixing[J]. IEEE Signal Processing Magazine, 2002:44-57.

[5] Bioucas-Dias J. M. et al. Hyperspectral Unmixing Overview: Geometrical, Statistical, and Sparse Regression-Based Approaches[J]. IEEE Journal of Selected Topics in Applied Earth Observations and Remote Sensing, 2012, 5(2): 354-379.

[6] Pati Y. C., Rezahfar R., Krishnaprasad P. Orthogonal matching pursuit: Recursive function approximation with applications to wavelet decomposition[C]. Asilomar Conf. Signals, Systems and Computing (ASSC), 2003. 1: 1-10.

[7] Chen S., D. Donoho, and M. Saunders. Atomic decomposition by basis pursuit[J]. SIAM Review, 2001, 43(1): 129-159.

[8] Rogge D. M., B. Rivard, J. Zhang, and J. Feng. Iterative spectral unmixing for optimizing per-pixel endmember sets[J]. IEEE Transactions on Geoscience and Remote Sensing, 2006, 44(12): 3725-3736.

[9] Bioucas-dias J. M., and M. Figueiredo. Alternating direction algorithms for constrained sparse regression application to hyperspectral unmixing[C]. WHISPERS'2010, Raykjavik, Iceland, 2010.

[10] Iordache M. D., Bioucas-Dias J. M., Plaza A. On the Use of Spectral Libraries to Perform Sparse Unmixing of Hyperspectral data[C]. IEEE GRSS Workshop Hyperspectral Image Signal Process.: Evolution in Remote Sensing (WHISPERS), 2010, 1:1-4. 
[11] Iordache M. D., Bioucas-Dias J. M., Plaza A. Sparse Unmixing of Hyperspectral Data[J]. IEEE Transactions on Geoscience and Remote Sensing, 2011, 49(6): 2014-2039.

[12] Iordache M. D., Bioucas-Dias J. M., Plaza A. Sparse Unmixing of Hyperspectral Data[J]. IEEE Transactions on Geoscience and Remote Sensing, 2011, 49(6): 2014-2039.

[13] Somers, B., G. P. Asner, L. Tits, P. Coppin. Endmember variability in Spectral Mixture Analysis: A review[J]. Remote Sensing of Environment, 2011, 115(7): 1603-1616.
[14] Yang ZY, et al. Blind Spectral Unmixing Based on Sparse Nonnegative Matrix Factorization[J]. IEEE Transactions on Image Processing, 2011, 20(4): 1112-1125.

[15] Chang C.I. et al. Maximum Orthogonal Subspace Projection Approach to Estimating the Number of Spectral Signal Sources in Hyperspectral Imagery[J]. IEEE Journal of Selected Topics in Signal Processing, 2011, 5(3): 504-520.

[16] Heinz, D. C., and C. I. Chang. Fully constrained least squares linear spectral mixture analysis method for material quantification in hyperspectral imagery[J]. IEEE Transactions on Geoscience and Remote Sensing, 2001, 39 (3): 529-545. 